\title{
1 Visual space curves before eye movements
}

2 Ifedayo-EmmanuEL Adeyefa-Olasupo ${ }^{1 *} \&$ Zixuan Xiao $^{4}$

$3 \quad{ }^{1}$ Department of Psychiatry and Psychotherapy, Charité - Universitätsmedizin Berlin

$4 \quad *$ Berlin School of Mind and Brain, Humboldt-Universität zu Berlin

$5 \uparrow$ Department of Neuroscience, Yale University

$6 \quad{ }^{4}$ Department of Psychology, Beijing Normal University

\section{ABSTRACT}

9 In most if not all experiments within cognitive and systems neuroscience, fixation is required before 10 the onset of an experimental trial. However, the term "fixation" is rather misleading since our eyes are 11 constantly moving. One type of transient miniature movements observed during fixation is commonly 12 referred to as fixational eye movements. Traditionally, and even in more recent experiments, the 13 inevitable effects of fixational eye movements on visual perception have been largely overlooked. To 14 date, no study has been able to provide an account of the extent to which fixational eye movements 15 actively mediate visual perception well before and around the time of a rapid eye movement (a saccade) 16 at retinotopic locations that constitute distinct retinotopic regions of visual space. Oculomotor planning 17 and performance effects associated with these transient shifts around the time of a saccade remain 18 equally unknown. Here, we systematically measured visual sensitivity in human subjects to visual 19 probes presented during fixation, well before and around the time of a saccade, at geometrically 20 symmetric retinotopic locations in the foveal, parafoveal and peripheral regions of visual space. We 21 observed transient local asymmetric visual sensitivity differences between these symmetric retinotopic 22 locations where none should be observed. Surprisingly, we also observed that saccade trajectories, 23 which are expected to travel along a linear path, routinely deviate along a curved path towards 24 orthogonal eccentric retinotopic locations. As such, we proposed a novel neurobiologically inspired 25 phenomenological force field model in which underlying attentional and oculomotor signals are 26 modulated by transient eccentric error signals that manifest as temporally overlapping forces and act 27 on retinotopic brain areas. These forces, which transiently shift putative population sensitivity towards 28 retinotopic foveal sites to support transient orthogonal fixational eye movements, and, shortly after, 29 along an axis orthogonal to the saccade direction towards a mislocalized peripheral site, succinctly 30 capture the essence of our perceptual and oculomotor observations. 


\section{INTRODUCTION}

34 The central $2^{\circ}$ of the visual field is extensively represented in the retina, the superior colliculus

35 (SC), and the primary visual cortex (V1), and is thus well suited for tasks that necessitate detailed 36 inspections of everyday visual objects (e.g., fruits, human faces, traffic signals). Under these 37 conditions, transient shifts in gaze, which are marked by fixational eye movements, are ubiquitous 38 (1). These transient shifts in gaze, which, as previous studies have shown, actively mediate visual 39 perception in general well before they occur, precede rapid eye movements, saccades (2-3). However, whether these transient shifts in gaze, once they occur, actively mediate visual perception well before and around the time of a saccade at retinotopic locations that constitute distinct retinotopic regions of visual space (i.e., the foveal, parafoveal or peripheral retinotopic regions) remains unknown (4-5). The oculomotor consequences associated with the onset of these transient shifts in gaze around the time of a saccade are also unknown.

Equally unclear is the extent to which early retinotopic representations that support local transient shifts in gaze during fixation influence peri-saccadic spatiotemporal retinotopic shifts. Specifically, recent behavioral and neurophysiological studies have suggested that around the time of a saccade, cells across retinotopic brain areas do not purely shift (or translate) from their current retinotopic locations of sensitivity to fixed future post-saccadic retinotopic locations of sensitivity as they primarily converge towards the target of the saccade $(6,7)$. Oddly enough, some of these studies have also reported convergent shifts orthogonal to the saccade direction, almost as if the location of the fixed saccade target is actively being ignored by the visual system (7-10). Unquestionably, these results, which report either purely translational (11-12) or symmetric and orthogonal convergent effects emerge from experiments that require fixation, during which transient shifts in gaze occur (1-5). Keeping in mind that impending fixational eye movements cause human subjects to erroneously perceive eccentric stimuli as being shifted away from their actual fixed locations (2), it is plausible that the extent to which retinotopic cells shift beyond the spatial extent of their fixed future post-saccadic retinotopic locations or converge along an axis that is orthogonal to the saccade direction could indeed be the result of retinotopic representations

60 which support transient shifts in gaze that occur well before a saccade - an account that has been 61 completely overlooked by previous translational and convergent studies. 
In addition to these unknowns, the neural computations and architecture that support spatial constancy - the visual system's ability to account for the incessant retinal disruptions that necessarily accompany saccades (13) - remain poorly understood. Recent studies have shown that retinal suppression signals triggered by fixational eye movements, which do not depend on corollary discharge commands originating from pre-motor brain areas (14), temporarily reduce visual sensitivity across visual space around the time of a saccade. It is therefore possible that attentional and oculomotor signals that support the allocation of more neural resources towards the foveal retina during fixation (15-20), at the expense of neural resources that would otherwise support intermediate eccentric retinotopic locations likely to contain retinal disruptions, are important for uncovering the neural computations and architecture underlying spatial constancy. Assuming these attentional and oculomotor signals play an important role, purely translational signals around the time of a saccade - which researchers once believed could explain spatial constancy $(13,21)$ but have been exposed by recent behavioral and electrophysiological studies as the result of coarse spatial sampling $(6,22)$ - would not be required for the visual system to achieve spatial constancy $(21)$.

\section{RESULTS}

To uncover how the onset and continual shifts in gaze during fixation actively influence (i) visual

80 sensitivity at retinotopic locations that constitute distinct retinotopic regions of visual space, (ii)

81 oculomotor planning and performance around the time of a saccade, (iii) peri-saccadic

82 spatiotemporal translational and convergent retinotopic shifts and (iv) the neural computations and

83 architecture that could support spatial constancy, we first assessed transient shifts in gaze after the

84 onset of a saccade target presented at a fixed retinotopic location during fixation and around the

85 time of a saccade. We later assessed saccade landing positions after subjects executed a saccade,

86 which informed us as to where subjects perceived the fixed saccade target to be located during

87 fixation. Following this, we assessed the transient consequences of visual sensitivity to visual

88 probes that were presented around the path of a saccade in the foveal, parafoveal, and peripheral

89 retinotopic regions of visual space during fixation and around the time of a saccade. In addition to

90 this, saccade trajectories were observed in order to assess whether the planning of a saccade and

91 its eventual execution along with observed transient changes in visual sensitivity share an 
92 underlying cause. Next, we investigated the rhythmicity that actively supported these transient

93 changes in visual sensitivity in order to assess whether they resemble known rhythmic signatures

94 that mediate neural activity across retinotopic brain areas during fixation and around the time of a

95 saccade. Finally, we proposed a novel neurobiologically inspired phenomenological force field

96 model that succinctly recapitulated transient changes in visual sensitivity at less (foveal) and more

97 eccentric (parafoveal) retinotopic of visual space.

98 In a dimly lit room, during fixation, head-fixed human subjects were randomly flashed

99 low-contrast probes (at a contrast level which the subject could detect with $50 \%$ accuracy) for

$10020 \mathrm{~ms}$ at 2.5 degrees of visual angle from the fixation dot (Experiment 1), from the midpoint (at an

101 eccentricity of $5^{\circ}$ ) between the fixation dot and the fixed saccade target (Experiment 2), or from

102 the fixed saccade target (Experiment 3), at tangential retinotopic locations (i.e., retinotopic

103 locations around the presumed path of a saccade, Fig. 1A), geometrically symmetric to one another

104 with respect to the radial axis (i.e., the presumed path along which subjects should plan their

105 impending saccade). Temporally, low contrast probes were flashed either during fixation or after

106 the extinguishment of the fixation dot, which served as the central oculomotor cue for subjects to

107 make a planned saccade towards the retinotopic location along which they perceived the fixed

108 saccade target to be positioned (See Methods, Fig. 1B). Subjects reported with a button-press

109 whether they were able to detect a flashed probe. To control for artificial restriction of attentional

110 and oculomotor resources along the tangential axes, probes were also isotropically flashed along

111 the radial axis. To then control for any false responses, in $25 \%$ of all experimental trials no probes

112 were flashed, which yielded a false alarm rate of $1.2 \%$ along the tangential axes. Indeed, because

113 of the aim of our study highlighted above, perceptual and oculomotor responses obtained in both

114 control conditions were discarded and are therefore not included in the main analyses reported

115 below. With respect to target onset, flashed probes fell within the time window of 0 to

$1161285 \mathrm{~ms}$ after target onset (Fig. 1B).

118 During fixation, the retinotopic location of the fixed saccade target appeared orthogonally 119 mislocalized, which reflects initial transient orthogonal shifts in gaze but does not reflect 120 later transient orthogonally compressed shifts in gaze.

122 Across experiments, after the onset of the fixed saccade target during fixation, as expected, we 123 observed transient shifts in gaze (1-5). However, specifically from 0 to $209 \mathrm{~ms}$ after the onset of 
124 the fixed saccade target, these transient shifts in gaze were unexpectedly averted away from the

125 retinotopic location of the fixed saccade target in the direction of the foveal tangential clockwise

126 fov $_{c w}$ retinotopic location (Fig. 2A, 5, 23). From 210ms to 820ms after the onset of the fixed

127 saccade target (Fig. 2B), we observed that these transient shifts in gaze continued to remain

128 eccentric but were compressed towards the foveal tangential counterclockwise fov ${ }_{c c w}$ retinotopic

129 location. This compression in gaze, in anticipation of a planned saccade, is in part in agreement

130 with previous reports of peri-saccadic compression of retinotopic receptive fields toward the

131 periphery, prior to the execution of a planned saccade $(7,24)$. The spatial distribution between

132 these marked transient shifts in gaze along the radial and tangential axes was statistically

133 significant $\left(\operatorname{rad}_{\text {shift }}, \mathrm{p}=4.0058 \mathrm{e}-36 ; \tan _{\text {shift }}, \mathrm{p}=0.0149\right)$. In all, these transient shifts in gaze

134 are in alignment with previous studies which report increases in neural activity in the posterior

135 parietal cortex (PPC) during transient shifts in gaze that were biased towards eccentric retinotopic

136 locations in visual space (25). Not surprisingly, the PPC has direct and independent connections

137 to the SC which is known to support the maintenance of fixation and, at the same time, initiate

138 transient shifts in gaze during fixation $(26,27)$. Across experiments, we observed that the saccade

139 landing position, from 0 to $50 \mathrm{~ms}$ after saccade offset (i.e., the moment the eye lands in the

140 periphery) was dramatically mislocalized towards the peripheral tangential clockwise peri $i_{c w}$

141 retinotopic location (Fig. 2C). Because the oculomotor system, as it plans an impending saccade,

142 must estimate the spatial extent of the eccentric saccade target that is concurrently being sampled

143 on the peripheral retina during fixation (28), this observation shows that subjects erroneously

144 perceived the fixed eccentric saccade target in the direction of the peri $i_{c w}$ retinotopic location

145 before the onset of the central oculomotor cue. This observation is in part in agreement with

146 previous reports of increases in neural activity in the PPC as the organism attempts to sample a

147 visual target of interest that is inherently difficult to track given the elusiveness of the visual target

148 (e.g., its eccentric spatial extent) or during a cued oculomotor task (29-30).

149 As expected, a larger variance was observed for the initial locus of gaze $\left(6.84 \mathrm{deg}^{2}\right)$ when

150 compared to the variance associated with the compressed locus of gaze $\left(2.12 \mathrm{deg}^{2}\right)$. As for the

151 variance associated with the saccade landing position, a larger variance (10 $\left.\mathrm{deg}^{2}\right)$ was observed

152 when compared to the loci of the gaze in the fovea (Fig. 2D). These results strongly suggest that

153 the orthogonal bias in the locus of the saccade landing position (more eccentric retinotopic regions)

154 erroneously continued to mirror the orthogonal bias we observed in the initial transient shifts in 
155 gaze within the fovea, even as gaze actively compressed orthogonally towards the fov $v_{c c w}$ 156 retinotopic location.

Transient orthogonal shifts in gaze curve the representation of visual space and, on the perceptual domain, correlate with local asymmetric differences in visual sensitivity at geometrically symmetric retinotopic locations.

We hypothesize that observed transient orthogonally biased shifts in gaze during fixation and the orthogonal mislocalization of the fixed saccade target during fixation is the result of a distorted retinotopic representation of visual space in which the foveal retinotopic region is represented along the radial axis and, inversely, eccentric retinotopic regions are erroneously represented along an axis that is orthogonal to the radial axis as it has been reported in previous neurophysiological studies $(7,31)$. This interpretation of our results implies that well before a saccade and, to an extent, before the execution of a saccade, the retinotopic representation of visual space erroneously includes an angular component, that is, visual space curves. From a functional perspective, since the retinotopic representation of eccentric retinotopic regions will not reflect active visual processing, as it is the case for the less eccentric retinotopic region, measured eccentric retinotopic locations (i.e., $\operatorname{para}_{c w}$ and the peri ${ }_{c w}$ retinotopic locations) tangentially positioned in the direction which reflect initial transient orthogonal shifts in gaze should demonstrate transient increases in visual sensitivity. Along the same lines, measured eccentric retinotopic locations (i.e., $\operatorname{para}_{c c w}$ and the $\operatorname{peri}_{c c w}$ retinotopic locations) tangentially positioned away from initial transient orthogonal shifts in gaze should demonstrate transient declines in visual sensitivity. With respect to the fovea, the measured retinotopic location (i.e., the fov $v_{c w}$ retinotopic location) tangentially positioned in the direction towards which gaze is actively being shifted should demonstrate transient increases in visual sensitivity, while the retinotopic location (i.e., the $f o v_{c w}$ retinotopic location) tangentially positioned away from the target retinotopic location of the impending transient shift in gaze should demonstrate transient declines in visual sensitivity. Conversely, if visual sensitivity does not demonstrate local transient asymmetric differences in visual sensitivity as we expect, then observed transient differences, assuming they will be observed, cannot be directly attributed to a curvilinear representation of visual space.

At geometrically symmetric retinotopic locations within distinct retinotopic regions of visual space, we observed local transient asymmetric differences in visual sensitivity (Fig. 2E-G). 
187 Specifically, in the foveal retinotopic region, when subjects began to orthogonally compress their

188 gaze towards the fov $_{c c w}$ retinotopic location, we observed transient but marked increases in visual 189 sensitivity at the $f o v_{c c w}$ retinotopic location (3), while at the $f o v_{c w}$ retinotopic location, we 190 observed transient but marked declines in visual sensitivity (Fig. 2E). In the parafoveal retinotopic 191 region, from $210 \mathrm{~ms}$ to $820 \mathrm{~ms}$ after the onset of the fixed target, we observed transient but marked 192 increases in visual sensitivity at the $\operatorname{para}_{c w}$ retinotopic location, while at the $p a r a_{c c w}$ retinotopic 193 location we observed marked declines in visual sensitivity (Fig. 2F). Finally, in the peripheral 194 retinotopic region, from $210 \mathrm{~ms}$ to $820 \mathrm{~ms}$ after the onset of the fixed target, we observed transient 195 but marked increases in visual sensitivity at the peri $i_{c w}$ retinotopic location, while at the peri $i_{c c w}$ 196 retinotopic location, we observed transient but marked declines in visual sensitivity (Fig. 2G).

\section{Peri-saccadic sensitivity hand-offs in the more eccentric retinotopic regions of visual space} take place around the same time orthogonal convergent effects are known to occur.

In the parafoveal retinotopic region, we also observed peri-saccadic visual sensitivity "hand-offs" between $\operatorname{para}_{c c w}$ and $\operatorname{para}_{c w}$ retinotopic locations. Specifically, 1014ms after the onset of the fixed saccade target (i.e., $70 \mathrm{~ms}$ before saccade onset), the $\operatorname{para}_{c c w}$ retinotopic location that once experienced transient declines in visual sensitivity begun to experience rapid increases in visual sensitivity, while the $\operatorname{para}_{c w}$ retinotopic location that once experienced transient increases in visual sensitivity began to experience rapid declines in visual sensitivity (Fig. 2F). Along the same lines, in the peripheral retinotopic region, we observed peri-saccadic hand-offs between peri $i_{c c w}$ and peri $_{c w}$ retinotopic locations. Specifically, 920ms after the onset of the fixed saccade target (i.e., $164 \mathrm{~ms}$ before saccade onset), the peri $_{c c w}$ retinotopic location that once experienced transient declines in visual sensitivity begun to experience rapid increases in visual sensitivity, while the

$211 \operatorname{peri}_{c w}$ retinotopic location that once experienced transient increases in visual sensitivity began to 212 experience rapid declines in visual sensitivity (Fig. 2G). As expected, in the foveal retinotopic 213 region, from $820 \mathrm{~ms}$ onwards after the onset of the fixed saccade target, we observed rapid global 214 declines in visual sensitivity at both foveal retinotopic locations (Fig. 2E). On one hand, these 215 global declines in visual sensitivity are in agreement with foveal suppression signals originating 216 from the retina to retinotopic brain areas before subjects execute a saccade (32). On the other hand, 217 observed peri-saccadic visual sensitivity hand-offs strongly suggest that, around the time subjects 218 began to prepare a saccade, eccentric retinotopic regions of visual space began to appear to subjects 
219 more radially. This means that retinotopic locations (i.e., $\operatorname{para}_{c c w}$ and peri $i_{c c w}$ retinotopic 220 locations) tangentially positioned in the direction towards which eccentric retinotopic 221 representation of visual space is being calibrated, experienced rapid increases in visual sensitivity, 222 while retinotopic locations (i.e., $\operatorname{para}_{c w}$ and peri $_{c w}$ retinotopic locations) tangentially positioned 223 away from the direction towards which eccentric retinotopic representation is being calibrated, 224 experienced rapid declines in visual sensitivity. These observed hand-offs in visual sensitivity, 225 along with the earlier observed fact that fixed saccade target can be orthogonally mislocalized 226 away from its actual retinotopic location during fixation strongly suggest that how quickly a 227 curvilinear visual space is calibrated by the visual system before saccade onset will fundamentally 228 determine (i) the extent to which cells in the extrastriate cortex will converge along an axis that is 229 orthogonal to the saccade direction $(7,31)$, and (ii) the extent to which human subjects 230 orthogonally mislocalize flash eccentric stimuli away from or towards the retinotopic location in 231 which the fixed saccade target is actually positioned $(32,33)$.

233 Saccades that are planned during fixation, expected to travel along the radial axis, deviate 234 along a curved path towards orthogonal eccentric retinotopic locations positioned beyond 235 the spatial extent of future post-saccadic retinotopic locations.

237 Following these observations on the perceptual domain, a natural question one might ask is 238 whether a curvilinear retinotopic representation of visual space accompanies any oculomotor 239 consequences, specifically around the time of a saccade. Keeping in mind that a curvilinear 240 representation of visual space predicts that eccentric retinotopic regions of visual space are 241 represented along an axis that is orthogonal to the radial axis, coupled with the fact that this 242 representation precedes and, in some cases, temporally overlaps with when subjects begin to plan 243 and prepare for an impending saccade (i.e., 150 to 200ms before saccade onset) (34, 35), we

244 predict that saccades on average will deviate along a curved path towards the peri $i_{c w}$ retinotopic 245 location - the retinotopic location towards which subjects on average mislocalized the fixed 246 saccade target (Fig. 2C). We further predict that because curved visual space is eventually 247 calibrated back towards $\operatorname{para}_{c c w}$ and peri $_{c c w}$ retinotopic locations, as the hand-offs in peri248 saccadic visual sensitivity observed in our perceptual experiences suggest, some saccades should 249 also curve towards the peri $i_{c c w}$ retinotopic location. Assuming our predictions are correct, this 250 would further strengthen the idea that the visual system is not exclusively spatially selective for 
future post-saccadic retinotopic locations that are uniquely parallel to the radial axis (i.e., the path saccades are expected to travel along, 36,37,38), but can actively select retinotopic locations that are positioned beyond these fixed post-saccadic retinotopic locations, for example, retinotopic locations orthogonal to the radial axis that convey task-relevant information $(21,22)$.

Aligned with this prediction, we observed that trajectories towards the periphery systemically deviated along a curvilinear path across experiments, at a rate of $73 \%$ towards the peri $_{c w}$ retinotopic location. In $27 \%$ of all trials, saccades were curved towards the peri $i_{c c w}$ retinotopic location. Specifically, when the saccade target was presented at a fixed azimuth angle of $0^{\circ}$, the trajectories were curved towards the peri $i_{c c w}$ retinotopic location at a rate of $91 \%$ with an average curvature of 0.22 in area/amp. Conversely, for the remaining trials (saccades curved towards the peri $_{c c w}$ retinotopic location), an average curvature of -0.80 in area/amp was observed. When the saccade target was presented at a fixed azimuth angle of $45^{\circ}$, the trajectories were curved towards the peri $_{c w}$ retinotopic location at a rate of $65 \%$ with an average curvature of 0.56 in area/amp, while for the remaining trials (saccades curved towards the peri $i_{c c w}$ retinotopic location), an average curvature of -0.74 in area/amp was observed. Finally, when the saccade target was presented at a fixed azimuth angle of $315^{\circ}$, trajectories were curved towards the retinotopic peri $_{c w}$ retinotopic location at a rate of $60 \%$ with an average curvature of 1.12 in area/amp, while for the remaining trials (saccades curved towards the retinotopic location peri $i_{c c w}$ location), an average curvature of -1.17 in area/amp was observed.

Delta-rhythmicity known to suspend neural resources and mediate neural activity in retinotopic brain areas also supports local asymmetric differences in visual sensitivity at geometrically symmetric retinotopic locations.

So far, we have provided an account of the functional correlates (i.e., the perceptual and oculomotor consequences) of curved visual space, which arise from transient orthogonal shifts in gaze during fixation. We posited that these transient orthogonal shifts occur because cells that support the more eccentric retinotopic regions of visual space are devoid of neural resources they need to correctly (or actively) process the visual world. This results in an erroneous representation of eccentric retinotopic regions along an axis that is orthogonal to the radial axis which (i) gives rise to asymmetric differences in visual sensitivity at geometrically symmetric retinotopic

282 locations at less and more eccentric retinotopic regions of visual space, and (ii) curves saccade 
trajectories expected to travel along a linear path. Indeed, these effects can also account for orthogonally biased translational and orthogonal convergent effects researchers have reported in previous studies. Here, we hypothesize that the neural resources these eccentric retinotopic cells once possessed are temporarily allocated toward orthogonal foveal sites (15-20), at and around the fov $v_{c w}$ retinotopic location, for example. To test this hypothesis, we investigated whether changes in visual sensitivity are uniquely supported by the same spectral signatures which suspend neural resources during cognitively demanding tasks (39) and which are known to mediate neural activity in brain areas that specifically support fixational eye movements and/or the planning and preparation of a saccade (e.g., frontal and parietal cortex, 6, 12, 25, 29, 30, 40). To this end, we transformed our detrended raw time-series visual sensitivity data into the frequency domain. The logic behind this investigation is as follows: if the changes in visual sensitivity we measured at retinotopic locations are supported by rhythms which are known to suspend the spreading of neural resources (with the exception of the $f o v_{c w}$ retinotopic location), then the spectral signature associated with these changes in visual sensitivity should strongly resemble the known rhythmic signatures (i.e., delta rhythms), especially at more eccentric retinotopic locations. Conversely, if the spectral signatures we observe do not recapitulate these known rhythmic signatures, then these transient changes cannot be directly attributed to the suspension of neural resources at the more eccentric retinotopic regions of visual space.

At the $f o v_{c c w}$ retinotopic location, we observed that these transient changes in visual sensitivity were actively mediated by rhythmicity in the delta frequency band at $\sim 2 \mathrm{~Hz}$. At the fov $v_{c w}$ retinotopic location, we observed that these transient changes in visual sensitivity were actively being mediated by rhythmicity at $\sim 3 \mathrm{~Hz}$. This higher rhythmicity is known to mediate the allocation of attentional signals that support visual sensitivity at suprathreshold levels (41-43). Furthermore, the spectral signature that mediated the fov $v_{c c w}$ retinotopic location was not locationspecific when compared to shuffled data sets $(\mathrm{p}=0.2280)$. Conversely, the spectral signature that supported the $f o v_{c w}$ retinotopic location was specific to that retinotopic location $(\mathrm{p}=0.0230)$. The $\operatorname{para}_{c w}, \operatorname{para}_{c w}, \operatorname{peri}_{c c w}$, and peri $i_{c c w}$ retinotopic locations also exhibited rhythmicity in the $\sim 2$

$310 \mathrm{~Hz}$ delta frequency band (Fig. 3C \& 3E). The spectral signature observed at these eccentric 311 clockwise and counterclockwise retinotopic locations were not location-specific $(\mathrm{p}=0.0915$, $312 \mathrm{p}=0.9725, \mathrm{p}=0.3179, \mathrm{p}=0.9725$ ) (Fig. 3D \& 3F). Furthermore, the phase of $\sim 2 \mathrm{~Hz}$ within para $_{c w}$ 313 and $\operatorname{para}_{c c w}$ retinotopic locations was consistently offset by approximately $201^{\circ}$, and even larger 314 offset of $290^{\circ}$ was observed at the more eccentric peri $i_{c w}$ and peri $i_{c c w}$ retinotopic locations (Fig. 
$3153 \mathrm{G} \& 3 \mathrm{H})$. This antiphase periodicity at $\sim 2 \mathrm{~Hz}$ further strengthens the idea of peri-saccadic visual

316 sensitivity hand-offs, suggesting that the retinotopic brain areas which support these geometrically

317 symmetric eccentric tangential retinotopic locations are synchronized by attentional dynamics at a

318 common frequency, but with location-specific phases (44).

\section{Neurobiologically inspired retinotopic lever under an inverse force field.}

322 Our spectral results suggest that observed transient changes in visual sensitivity may indeed be the consequences of signals that suspend attentional resources at the more eccentric retinotopic

324 locations and directly enhance active foveal visual processing, namely at the fov $v_{c w}$ retinotopic

325 location. Remarkably, at this retinotopic location, rhythmic visual sensitivity patterns were

326 uniquely spatially selective. It is also this location towards which subjects initially shifted their

327 gaze after the onset of the fixed saccade target. Finally, it is from this very same retinotopic

328 location that attentional resources must be shifted towards the $f o v_{c c w}$ retinotopic location in order

329 to enhance visual sensitivity there $(3,7,24)$.

330 With these insights from our spectral investigations, along with the fact that population 331 receptive fields (pRFs) across retinotopic brain areas can transiently shift beyond the spatial extent 332 of their classical receptive fields, we developed a simple but powerful novel neurobiologically 333 inspired phenomenological force field model (See Methods). From an architectural standpoint, our 334 model includes retinotopic pRFs that tile foveal, parafoveal, and peripheral retinotopic regions of 335 visual space. With the context of our model, these pRFs can be understood as spring-loaded 336 sensors. Another layer in our model is a force field, which is composed of forces, exerted by 337 corresponding time-varying masses that act on pRFs. Finally, the third layer of our model consists 338 of retinotopic population elastic fields ( $\mathrm{pEFs}$ ) which all pRFs possess in order to always maintain 339 retinotopic organization during transient retinotopic shifts in visual space (e.g., Fig 4A). 340 Functionally, at its core, our model assumes that underlying neural (i.e., attentional and 341 oculomotor) signals, are actively modulated by transient eccentric error signals $(25,29,30)$. The 342 summation of these signals temporarily suspends neural resources at the more eccentric retinotopic 343 regions of visual space which causes the visual system to erroneously represent these regions along 344 an axis that is orthogonal to the radial axis while enhancing active visual processing at tangential 345 foveal retinotopic locations (20). This gives rise to temporally overlapping input and output forces 346 exerted by corresponding time varying masses positioned at fov $c c w$ and peri $i_{c w}$ retinotopic 
locations respectively (Fig. 4B \& 4E), which then act upon a "retinotopic lever". Under an inverse

348 force field ( $\varepsilon=-0.55$, see Methods), input and output forces transiently shift pRFs along an axis

349 that is orthogonal to the radial axis and thereby shape population density (putative population

350 sensitivity) at a sampled retinotopic location in time (Fig. 4A, Fig. 4D, and Extended Data Fig. 1-

$3512)$.

352

Spatio-temporal retinotopic shifts that curve visual space under an inverse-distance rule explain local asymmetric transient differences in visual sensitivity at geometrically symmetric locations in less eccentric and more eccentric retinotopic regions.

As far as the foveal simulations, attentional and oculomotor signals modulated by transient eccentric error signals give rise to an input force that is preparing to act on the input arm of a "retinotopic lever" which causes pRFs to orthogonally shift in the direction of the fov $v_{c w}$ retinotopic location and later compress towards the $f o v_{c c w}$ retinotopic location. A compressed input force causes the lever to pivot on its "foveal fulcrum" along a curvilinear path thereby providing a transient neural advantage at the $f o v_{c c w}$ retinotopic location. To always maintain retinotopic organization, $\mathrm{pRFs}$ that tile the more eccentric retinotopic regions in visual space are initially restricted from over-translating towards the foveal $f o v_{c w}$ retinotopic location by their pEFs, which remain mirroring the onset location of the input force before a full compression occurs. For the sake of simplicity, foveal simulations began from the point in time when pRFs mirrored the initial transient orthogonal shift in gaze (translational output force), followed by late transient compressive shift in gaze towards the fov $v_{c c w}$ retinotopic location (compressed input force) (Fig. 4B). Under these input and output forces, as expected, simulation results in Fig. 4C show that putative population sensitivity recapitulated signatures in transient changes in visual sensitivity in the less eccentric (foveal) retinotopic regions of visual space (Fig. 2E). Assuming that retinotopic cells are not mediated by transient eccentric error signals (i.e., retinotopic 373 representation does not include a curvilinear component), translational shifts researchers once 374 thought anticipated retinal image disruptions saccades accompany (11-13) should have yielded no 375 difference in putative population sensitivity (Fig. 4C, dotted marker) in comparison to the transient 376 changes in visual sensitivity we observed in our behavioral experiments (Fig. 2E). Similarly, 377 symmetric convergent retinotopic shifts towards a fixed peripheral site (Fig. 4C, thin markers, 6) 378 cannot explain observed transient changes in visual sensitivity. 
As far as the eccentric simulations (Fig. 4D), after a fully compressed input force, pRFs moved further towards the $f o v_{c c w}$ retinotopic location in proportion to the mislocalization of the fixed saccade target. Cells across retinotopic brain areas can receive both convergent and translational signals around the time of a saccade $(6,12)$. To this end, pRFs later converged around an emerging output mass which exceeded the magnitude of the initial transient orthogonal shift in gaze and approximated the retinotopic location of the mislocalized target (Fig. 2A \& 2C). This is immediately followed by the onset of a translational force proportional to the magnitude of a saccade orthogonal to the radial axis towards the peri $i_{c w}$ retinotopic location (Fig. 4E). Indeed, under these three forces, especially when the forces that correspond to error modulated convergent signals emerge, a neural advantage at eccentric clockwise retinotopic locations should occur. As expected, Fig. 4F shows that putative population sensitivity was able to recapitulate transient visual sensitivity signatures at parafoveal retinotopic locations (Fig. 2F). In all, as expected, a purely translational or symmetric convergent retinotopic shifts cannot explain observed transient changes in visual sensitivity.

\section{DICUSSION}

There is now a growing consensus that fixational eye movements actively mediate visual perception well before they occur (e.g., 2, 3). For example, in 2011, Hafed found that before the onset of fixational eye movements the retinotopic representation of visual space compresses towards the site of the impending fixational eye movement. This peri-mircosaccadic compression of visual space causes human subjects to erroneously perceive foveal stimuli as more eccentric and conversely eccentric stimuli as more foveal. However, while some of the effects of fixational eye movements on visual perception well before and even after they occur have been uncovered (e.g., 5), the extent to which transient shifts in gaze actively mediate visual sensitivity at retinotopic locations that constitute distinct retinotopic regions of visual space remained unknown. Equally unaccounted for is the influence of transient shifts in gaze on oculomotor planning and performance around the time of a saccade. To provide an account from a functional perspective, we systematically measured the consequences of transient shifts in gaze during fixation on visual sensitivity at geometrically symmetric retinotopic locations in the foveal, parafoveal, and peripheral regions of visual space. Next, we assessed how these transient shifts in gaze mediate the planning and execution of a saccade trajectory. We show for the first time that these transient 
411 shifts in gaze give rise to local asymmetric differences in visual sensitivity at geometrically

412 symmetric retinotopic locations, while saccade trajectories deviate along a curvilinear path 413 towards an orthogonal mislocalized peripheral site (Fig. 2E \& 2H). We later constructed a 414 phenomenological force field model in which we proposed that transient shifts in gaze are 415 underlined by attentional and oculomotor signals that are actively modulated by transient eccentric 416 error signals. The summation of these signals manifests as temporally overlapping forces which 417 act on cells across retinotopic brain areas and thereby modulate their population sensitivity 418 equivalent to changes in visual sensitivity in our psychophysical experiments. Putative population 419 sensitivity signatures recapitulated local asymmetric differences in visual sensitivity we observed 420 at geometrically symmetric retinotopic locations in less and more eccentric retinotopic regions of 421 visual space (Fig. 2E \& 2F, Fig. 4C, and Fig. 4F).

422 Apart from uncovering the effects of transient shifts in gaze on visual sensitivity and 423 saccade trajectory, our behavioral and simulation results provide three fundamental insights into 424 how early retinotopic representation actively modulates peri-saccadic representation. First, peri425 saccadic representation of visual space reflects an overriding influence of attentional and 426 oculomotor signals which are actively modulated by transient eccentric error signals and are not 427 the result of purely translational or symmetrically convergent signals, as suggested by previous 428 studies $(6,11,12,21)$. Indeed, the presence of or the gating off of these eccentric error signals 429 seems to determine (i) whether flashed stimuli will be orthogonally mislocaized away from or 430 towards a fixed saccade target (Fig. 2F \& 2G, 32,33), (ii.) whether cells in the extrastriate cortex 431 will align with or shift along an axis that is orthogonal to the radial axis $(7,8)$ and (iii) the extent 432 to which peri-saccadic retinotopic shifts will translate towards future post-saccadic retinotopic 433 locations or shift towards tangential retinotopic locations positioned beyond the spatial extent of 434 future post-saccadic sites (Fig. 2H, 22). Second, keeping in mind that the purely translational (or 435 forward) account of spatial constancy which researchers once thought could explain this retinal 436 phenomenon $(13,21)$ has been challenged on methodological grounds $(6,7,24)$, our results go 437 one step further and lead to a proposal of a novel mechanism that can explain spatial constancy. 438 Specifically, our results strongly suggest that well before a saccade and, to some extent, around 439 the time of a saccade, the visual system transiently allocates visual resources that would otherwise 440 support active visual processing at intermediate eccentric retinotopic locations towards the fovea, 441 resources, which are then then used to further enhance active visual processing at specific foveal 442 retinotopic locations. This results in a drastic diminishing of active visual processing at 
443 intermediate eccentric retinotopic regions of visual space. Furthermore, because peri-saccadic

444 visual resources spread at a glacial pace $(36,45)$, we posit that resources which eventually revert

445 back towards intermediate eccentric retinotopic locations arrive well after the execution of a rapid 446 movement towards the periphery (46). As opposed to translating visual resources to fixed post447 saccadic future retinotopic locations, we posit that the visual system transiently shifts and 448 compresses resources backwards (i.e., in the opposite direction of the fixed saccade target, 5, 23), 449 away from intermediate retinotopic locations likely to contain retinal disruptions. Because of this 450 difference in speed, that is, between eye movement during a saccade and the spread of eccentric 451 peri-saccadic visual resources to their equilibrium positions, our perception of the visual world can 452 escape the retinal disruptions that necessarily accompany rapid eye movements. Finally, the neural 453 computations that support this retinotopic representation well before and around the time of a 454 saccade obey an inverse-distance rule, while the neural architecture that mediates spatiotemporal 455 retinotopic shifts pRFs undergo - which give rise to this representation - includes pEFs.

456 In all, the observed transient shifts in gaze during fixation remain a curious point, since 457 they are directed away from the fixed saccade target. However, considering that humans were once 458 preys, it is possible that these miniature shifts away from the visual target of interest reflect an 459 evolutionarily conserved visual oculomotor avoidance or escape strategy the mammalian visual 460 system used to track a distal target (e.g., conspecific, predator) from a safe distance (e.g., corner 461 of the eye), later followed by a calculated movement (e.g., saccade) towards the retinotopic 462 location previously avoided (47-49).

\section{AUTHOR CONTRUBITIONS}

466 EL conceptualized the project. EL collected the data. EL preprocessed the behavioral data with the 467 assistance of XZ. EL analyzed the behavioral data. EL developed the computational model. EL implemented the model. EL performed the model simulations. EL wrote the manuscript.

\section{ACKNOWLEDGEMENTS}

471 We would like to thank D. P. Melcher and S. Wirth for comments on the manuscript and B. 472 Wichterlová for editing the manuscript. We would also like to thank A. S. Nandy and M. Scudder 473 for their guidance on the experimental design and implementation. 
476 Authors has no competing financial interest.

\section{DATA AVAILABILITY}

479 The data that supports this study is available upon reasonable request.

CODE AVAILABILITY

482 The custom Matlab code that supports the figures reported in this study is available upon

483 reasonable request.

\section{SUPPLEMENTAL TEXT}

Fig 1. Behavioural protocol. (A) Markers in the illustration of visual space show all retinotopic

487 locations at which a probe was flashed before and after the deployment of attention, saccade 488 planning, and, finally, the execution of a saccade. Probes of interest, presented along the tangential 489 axes, are conspicuously highlighted in open circle markers. (B) Temporal sequence of a valid trial 490 across experiments with respect to target onset.

492 Fig 2. Behavioural data. (A-D) Probability density map of foveal gaze and saccade landing was 493 calculated using a bivariate gaussian kernel along with their bivariate contour ellipse area estimates 494 for each probability density map. Error estimates are standard error of means across observers. (E495 G) Shifts in visual sensitivity along tangential axes with respect to target onset; error estimates 496 were calculated using a 20-fold jackknife procedure. (H) Mean saccade trajectory deviation for 497 horizontal and oblique saccades across experiments. Positive values on the ordinate denote 
498 trajectory deviation towards a clockwise location (cw), while negative values denote trajectory

499 deviation towards a counterclockwise location (ccw)

501 Fig 3. Frequency domain representation of visual sensitivity and cross-location phase

502 coherence estimates. (A-F) Representation of raw detrended visual sensitivity at less and more

503 eccentric locations in visual space. Shuffled ( $\mathrm{n}=1000$, dashed line) versus unshuffled (solid line)

504 data set were used to compute location-specific spectral signatures at a $p<0.05$. (G-H) For

505 parafoveal and peripheral clockwise and counterclockwise locations a cross-location phase

506 coherency analysis was performed.

508 Fig 4. Model architecture and neurobiological force field with simulated putative

509 population sensitivity. (A) For illustration purposes, a single eccentricity dependent population

510 receptive field (pRF) within a retinotopic field is highlighted in pink. A pRF is defined by its

511 center, size, and an eccentric dependent population elastic field (pEF). (B) MGa denotes gaze

512 transitional signals modelled as a virtual mass at infinity located in the direction of an upper

513 tangential location approximating the initial shift in gaze. MF denotes compressive signals

514 modelled as a varying mass located at a lower tangential location in the fovea. (C) Simulation

515 results in the foveal region when a pRF is under the influence of a resultant force exerted by (i)

$516 \mathrm{MF}$ and MGa (curved space, thick marker) (ii) MC (convergent signals modelled as a varying

517 mass at a stationary peripheral site; see Methods) and (iii) MT (saccade transitional signals

518 modelled as a virtual mass at infinity located in the direction of a straight saccade; see Methods).

519 (D) A pRF bounded by pEF is highlighted in pink for parafoveal simulations. (E) MG $\beta$ denotes

520 gaze translational signals modelled as a virtual mass at infinity located in the direction of a 
521 downward shifts in gaze, MM denotes a varying mass at a mislocalized peripheral target, and

522 MS denotes the curved transitional signals modelled as a virtual mass at infinity located in the

523 direction of a curvilinear saccade. (F) Curved, convergent and translational simulation results.

525 Extended Data Fig. 1 Spatiotemporal retinotopic shifts for foveal simulations. (A)

526 hexagonal retinotopic field spanning the entire visual field. Retinotopic shifts are produced by

527 the perturbation of pRFs under an inverse force field in discrete time increments of 1ms by the

528 combination of external forces exerted by $(\mathbf{B})$ corresponding masses $\left(\mathrm{M}_{\mathrm{G} \alpha}+\mathrm{M}_{\mathrm{F}}\right)$.

\section{Extended Data Fig. 2 Spatiotemporal retinotopic shifts for parafoveal stimulations. (A)}

531 Retinotopic shifts when the combination of external forces is exerted by (B) corresponding

$532 \operatorname{masses}\left(\mathrm{M}_{\mathrm{G} \beta}+\mathrm{M}_{\mathrm{M}}+\mathrm{M}_{\mathrm{S}}\right)$.

\section{METHODS}

\section{Human subjects}

536 Six consenting human subjects (5 females and 1 male; 21-26 years old) with corrected-to-normal

537 visual acuity participated in the three experiments reported as it is standard within the field of 538 research. Three of the six subjects participated in the foveal and parafoveal experiments. The 539 remaining three subjects participated in the peripheral experiment. The Yale Ethics Review board 540 approved the protocol of these experiments and the storage of the data that was collected. 
543 Fig. 1 summarizes the protocol used across experiments. In a dimly lit room, stimuli were

544 presented on a gamma corrected display with a spatial resolution of $1400 \mathrm{x} 1050$ pixels and a mean

545 luminance of $38 \mathrm{~cd} / \mathrm{m}^{2}$. Eye movements were recorded using an infrared video-based eye-tracker

546 sampled at $1 \mathrm{kHz}$ (I-Scan Inc., Woburn, MA). While tightly head fixed using a head rest and a

547 customized bite bar, subjects at the onset of each trial acquired fixation for 300ms. Fixation within

548 this temporal window was spatially aligned with the fixation point ( $0.5 \mathrm{dva}$ in diameter $)$ using a

549 custom-developed 9-point eye-calibration procedure in Picto. Next, depending on the subject, a

550 saccade target was presented at an eccentricity of $10^{\circ}$ and at azimuth angles of $0^{\circ}$ (for $s_{1}$ ), $45^{\circ}$ (for

$551 s_{2}$ ) and $315^{\circ}$ (for $s_{3}$ ) (Fig. 1A). Following target onset, fixation was maintained for another 300-

$552600 \mathrm{~ms}$ within 2 dva fixation tolerance window, at the end of which the fixation spot was

553 extinguished, which served as the central motor cue for subjects to make an eye movement towards

554 the saccade target. For another $500 \mathrm{~ms}$ after the central motor cue, the saccade target remained

555 visible. Finally, a low contrast probe (at a contrast level which the subject could detect with $50 \%$

556 accuracy) were isotropically flashed, depending on the experiment, for $20 \mathrm{~ms}$ either along the radial

557 or tangential axis at a random time during the $300-600 \mathrm{~ms}$ window between the target onset and

558 the central motor cue ( $25 \%$ of trials) or at a random time between $0-340 \mathrm{~ms}$ after the central motor

559 cue (50\% of the trials). On the remaining $25 \%$ (which yielded a false alarm rate of $1.2 \%$ ), no

560 probes were presented. All three conditions were randomly interleaved across trials. With respect

561 to target onset, the presentation of flashed probes fell within a time window of $0-1285 \mathrm{~ms}$ after

562 target onset (Fig. 1B). Subject was asked to report, using a push button, whether they were able to

563 detect the flashed probe. While the responses along the radial axis were not analyzed, the

564 presentation of these probes was crucial in controlling for any response biases that may have

565 emerged along the tangential axes. To ensure that attentional and oculomotor resources was not 
566 being driven by an asymmetric stimuli configuration as it is the case in previous studies, no

567 secondary salient spatial cue was presented. Along the tangential axes, a total of $\sim 6010$ trials were

568 collected across experiments, with a minimum of 650 trials per subject.

$570 \quad$ Statistical analyses

571 To investigate gaze dynamics, probability density maps and variance estimates were calculated 572 using median filtered edge preserving fixational data, which was rotated to an angle of $0^{\circ}$ across 573 target locations. Next, I calculated a gaze probability density map for the foveal and periphery 574 regions using a bivariate Gaussian kernel (50). The estimated locus of gaze across subjects was 575 defined as the peak of each probability density map (i.e., the yellow contour lines). To compare 576 the distribution of gaze in the fovea during the 0 to $209 \mathrm{~ms}$ window after target onset versus 210 to $577820 \mathrm{~ms}$ window after target onset, a Mann-Whitney U statistic was calculated at $\mathrm{p}$ value less than 578 0.05. Further, variance in gaze was calculated using a bivariate Contour Ellipse Area (BCEA) 579 approach which included $68 \%$ of fixational data around the mean (51). Accompanying error bars 580 were obtained which denote the standard error of the mean variance across subjects. To investigate 581 the extent saccade trajectories were curved across experiments at each saccade location. Horizontal 582 and vertical eye positions, with the time window starting point constituting the moment fixation 583 was maintained to the moment the eye landed in the periphery, was isolated. Next to obtain a 584 measurement of curvature, the curved area that emerged from the eye position during this time 585 window was calculated. To then obtain a ratio measure of curvature per unit amplitude, this area 586 was divided by the amplitude of the saccade (52). Accompanying calculated error bars denote the 587 standard error of the mean at each target location across experiments. To investigate normalized 588 changes in visual sensitivity across subjects, I first binned each subject's percent correct responses 
589 for the foveal (Exp. 1), parafoveal (Exp. 2), and peripheral (Exp. 3) experiments, using a 15ms

590 sliding window that was moved in $45 \mathrm{~ms}$ increments. Next, I obtained each subject's raw visual

591 sensitivity, using a 20-fold jackknife procedure by estimating sensitivity from $95 \%$ of the data.

592 This was repeated 20 times, each time leaving out $5 \%$ of the data. This produced a mean and an

593 error estimate of visual sensitivity at each tangential location for each experiment across subjects

594 (53). To control for any eccentric-dependent effect on raw visual sensitivity, these traces were

595 normalized by the average sensitivity over the initial $100 \mathrm{~ms}$ in the data. To investigate frequency

596 domain and cross-location phase coherence dynamics, a fast Fourier transform (FFT) was applied

597 to the detrended raw visual sensitivity data. Next, we computed the mean of the spectral signatures

598 and corresponding standard error of the mean across subjects. To than assess whether the observed

599 periodicities were location specific, we randomly shuffled $(n=1000)$ the probe location identities

600 across trials and calculated the sensitivity function and periodicity of the shuffled data using the

601 same procedures noted above. A two tailed paired-sample t-test at p-values of 0.05 was calculated

602 to assess the significance between shuffled and non-shuffled data. Finally, for the cross-location

603 phase coherence analysis, we applied an approach to the shuffled and non-shuffled detrended data

604 that is commonly used in assessing the underlying coherence in neural data (54). I then projected

605 the difference between phase angles of each subject between counterclockwise (ccw) and 606 clockwise (cw) locations and then calculated the resultant vector across subjects.

\section{Novel computational framework - Retinotopic mechanics}

609 The underlying architecture of this simple mechanical model is composed of a retinotopic field $610\left(\phi_{r}\right)$ and a retinotopic lever which is inspired by a neurobiological force field. The retinotopic field 611 is a two-dimensional overlapping hexagonal grid of eccentricity-dependent receptive fields $-\mathrm{RF}_{\mathrm{i}}$ 
612 - that tiled the foveal, parafoveal and peripheral regions in visual space (Extended Fig. 1-2). Each

$613 \mathrm{RF}_{\mathrm{i}}$ was defined by three parameters: (a) $p_{i}$, the spatial extent of the $\operatorname{RF}$ center $\left(x_{i}, y_{i}\right)$ at $t_{0}$ (when

614 there is no resultant force acting on the $\phi_{r}$ ). $p_{i}$ determines the eccentricity $-e_{i}$ of $\mathrm{RF}_{\mathrm{i}}$, (b) $s_{i}$, the

615 radius of $\mathrm{RF}_{\mathrm{i}}$ is proportional to $e_{i}$, and (c) the movement extent $\left(M_{\max }\right)$ of $\mathrm{RF}_{\mathrm{i}}$ is its $\mathrm{EF}_{\mathrm{i}}$ which is

616 also proportional to $e_{i} . \phi_{r}$ emerged from attentional and oculomotor signals actively modulated by

617 transient eccentric error signals that act as input and output forces of retinotopic lever in this field,

618 and transiently perturb a constellation of population receptive fields from equilibrium and thereby

619 modulate their putative population sensitivity (density readouts) as a function of space and time.

620 For simulations at the less eccentric ("foveal"), the resultant force $\left(\overrightarrow{\mathrm{F}_{\mathrm{R} 1}}\right)$ included an input and

621 output force $\left(\overrightarrow{F_{I}}\right.$, and $\left.\overrightarrow{F_{O}}\right)$ and an internal force $\left(\overrightarrow{F_{E}}\right)$ which $R F_{i}$ at $t_{0+n}$ experiences:

where $\mathrm{M}_{\mathrm{G} \alpha}$ and $\mathrm{M}_{\mathrm{F}}$ are the virtual mass at infinity in the upward direction which approximates an initial shift in gaze and a foveal mass located at a lower tangential foveal retinotopic site which

approximates a compressed shift in gaze respectively. $\overrightarrow{\mathrm{U}_{\mathrm{M}_{\mathrm{G} \alpha}}}$ and $\overrightarrow{\mathrm{U}_{\mathrm{M}_{\mathrm{F}}}}$ are the unit vectors in the

632 direction of the virtual mass and the foveal mass respectively. $|G \alpha|$ is the magnitude in gaze

633 towards the upper tangential foveal retinotopic site, while $R_{\beta}$ is the spatial difference between $R F_{i}$

634 and $\mathrm{M}_{\mathrm{F}}$ raised to an epsilon scalar $-\varepsilon$. The value of $\varepsilon$, depending on the retinotopic mechanics

635 that best explains the phenomenon in question, can either be consistent with a proportional-

636 distance rule (i.e., $>0$ ) or an inverse-distance rule (i.e., $<0)$. Further, $\mathrm{k}_{\mathrm{I}}$ was obtained by calculating 
637 the average of $\mathrm{R}_{\beta 0}^{\varepsilon}$ across $\mathrm{RF}_{\mathrm{i}}$ 's and then taking its reciprocal $\frac{1}{\left(\mathrm{R}_{\beta 0}^{\varepsilon}\right)}$, while $\overrightarrow{\mathrm{F}_{\mathrm{E}}}$ is proportional to

638 the movement of $R F_{i}$. For the more eccentric ("parafoveal") simulations, the resultant force $\left(\overrightarrow{F_{R 2}}\right)$

639 included an input, output, an additional orthogonally biased translational force $\left(\overrightarrow{\mathrm{F}_{\mathrm{I}}}, \overrightarrow{\mathrm{F}_{\mathrm{O}}}\right.$ and $\left.\overrightarrow{\mathrm{F}_{\mathrm{T}}}\right)$

640 and an internal force $\left(\overrightarrow{F_{E}}\right)$ which $R F_{i}$ at $t_{0+n}$ experiences:

641

642

643

644

645

646

647

648

649

650

652

653

654

655

656

657

658

659

660

661

$$
\overrightarrow{\mathrm{F}_{\mathrm{I}}}=\overrightarrow{\mathrm{U}_{\mathrm{M}_{\beta}}} \cdot \mathrm{M}_{\mathrm{G} \beta} \cdot|\mathrm{G} \beta|
$$

$$
\begin{gathered}
\overrightarrow{\mathrm{F}_{\mathrm{O}}}=\overrightarrow{\mathrm{U}_{\mathrm{M}_{\mathrm{M}}}} \cdot \mathrm{M}_{\mathrm{M}} \cdot \mathrm{R}_{\mu}^{\varepsilon} \\
\overrightarrow{\mathrm{F}_{\mathrm{T}}}=\overrightarrow{\mathrm{U}_{\mathrm{M}_{\mathrm{S}}}} \cdot \mathrm{M}_{\mathrm{S}} \cdot|\mathrm{S}| \\
\overrightarrow{\mathrm{F}_{\mathrm{R} 2}}=\overrightarrow{\mathrm{F}_{\mathrm{O}}} \cdot \mathrm{k}_{\mathrm{O}}+\overrightarrow{\mathrm{F}_{\mathrm{I}}}+\overrightarrow{\mathrm{F}_{\mathrm{T}}}+\overrightarrow{\mathrm{F}_{\mathrm{E}}}
\end{gathered}
$$

where $\mathrm{M}_{\mathrm{G} \beta}$ and $\mathrm{M}_{\mathrm{M}}$ are the virtual mass at infinity in the direction towards lower foveal tangential retinotopic site which approximates a complete compression of gaze and the mislocalized mass located at a peripheral retinotopic site that approximates a mislocalized saccade target respectively. $\overrightarrow{\mathrm{U}_{\mathrm{M}_{\mathrm{G} \beta}}}$ and $\overrightarrow{\mathrm{U}_{\mathrm{M}_{\mathrm{M}}}}$ are the unit vectors in the direction of the virtual mass and the mislocalized peripheral mass respectively. $|\mathrm{G} \beta|$ is the magnitude of a fully compressed gaze which is roughly proportional to the magnitude of a mislocalized target. $R_{\mu}$ is the spatial difference between $\mathrm{RF}_{\mathrm{i}}$ and $\mathrm{M}_{\mathrm{M}}$ raised to $\varepsilon$, while $|\mathrm{S}|$ approximates the magnitude of an impending saccade. The same calculation used to obtain $\mathrm{k}_{\mathrm{I}}$ was used to obtain $\mathrm{k}_{\mathrm{O}}$, however in this case, the average of $\mathrm{R}_{\mu 0}^{\varepsilon}$ across $\mathrm{RF}_{\mathrm{i}}$ 's was used. Depending on the stimulation, a time varying resultant force $\left(\overrightarrow{\mathrm{F}_{\mathrm{R} 1}}\right.$ or $\left.\overrightarrow{\mathrm{F}_{\mathrm{R} 2}}\right)$ perturbed each $\mathrm{RF}_{\mathrm{i}}$, which produced spatiotemporal retinotopic displacements (Extended Data Fig. 1-2). The population movements of the constellations of $\mathrm{RF}_{\mathrm{i}}$ 's manifested in time-varying modulation of raw density, modeling each $\mathrm{RF}_{\mathrm{i}}$ as a bivariate gaussian kernel function $G(55)$. A 
662 probability density estimate (or putative population sensitivity readouts), $\hat{f}$, was obtained using

663 the following equation:

664

665

666

$$
\begin{gathered}
G(u)=\frac{1}{\sqrt{2 \pi}} e^{-\frac{1}{2} u^{\mathrm{T}} u} \\
\hat{f}(\boldsymbol{x}, \mathbf{B})=\frac{1}{k} \sum_{\mathrm{i}=1}^{\mathrm{k}} \mathrm{G}_{\mathbf{B}}\left(\left|\boldsymbol{x}-\boldsymbol{x}_{\boldsymbol{i}}\right|\right)
\end{gathered}
$$

667

Finally, population sensitivity readouts were later normalized using the same approach used to normalize the raw visual sensitivity traces.

\section{REFERENCES}

1. Ratliff F, Riggs LA. Involuntary motions of the eye during monocular fixation. $J$ Exp Psychol. 40:687-701. (1950).

2. Hafed, Z. M. Alteration of visual perception prior to microsaccades. Neuron. 77, 775786. (2013).

3. Shelchkova N. Poletti M. Modulations of foveal vision associated with microsaccade preparation. Proc. Natl. Acad. Sci. USA 117: 11178-11183. (2020).

4. Chen, C. Y., Ignashchenkova, A., Thier, P., and Hafed, Z. M. Neuronal response gain enhancement prior to microsaccades. Curr. Biol. 25, 2065-2074. (2015).

5. Tian, X., Yoshida, M., Hafed, ZM. A microsaccadic account of attentional capture and inhibition of return in Posner cueing. Front Syst Neurosci. 10:23. (2016). 
7. Tolias, A. S., Moore, T., Smirnakis, S. M., Tehovnik, E. J., Siapas, A. G., Schiller, P. H. Eye movements modulate visual receptive fields of V4 neurons. Neuron, 29(3), 757-767. (2001).

8. Connor, C. E., Gallant, J. L., Preddie, D. C., Van Essen, D. C. Responses in area V4 depend on the spatial relationship between stimulus and attention. Journal of Neurophysiology, 75(3), 1306-1308. (1996).

9. Kaiser, M., Lappe M. Perisaccadic mislocalization orthogonal to saccade direction. Neuron, 22;41(2), 293-300. (2004).

10. Zimmermann, E., Morrone, M. C., Burr, D. C. Visual mislocalization during saccades sequences. Exp Brain Res, 233(2):577-585. (2014).

11. Duhamel, J. R., Colby, C. L., Goldberg, M. E. The updating of the representation of visual space in parietal cortex by intended eye movements. Science, 255(5040), 90-92. (1992).

12. Umeno, M.M., Goldberg, M.E. Spatial processing in the monkey frontal eye field. I. Predictive visual responses. J Neurophysiol, 78:1373-138. (1997).

13. Wurtz R. H. Neuronal mechanisms of visual stability. Vision research, 48(20), 2070 2089. (2008). saccadic suppression starts in the retina. Nat. Commun. 11, 1977. (2020).

16. Richard, A., Churan, J., Guitton, DE., Pack, CC. The geometry of perisaccadic visual perception. J Neurosci, 29: $10160-10170$, (2009).

15. Hamker, FH., Zirnsak, M., Calow, D., Lappe, M. The peri-saccadic perception of objects and space. PLoS Comput Biol, 4: e31. (2008).

17. VanRullen, R. A. simple translation in cortical log-coordinates may account for the pattern of saccadic localization errors. Biol Cybern, 91: 131-137. (2004). 
18. Zirnsak, M., Lappe, M., Hamker, FH. The spatial distribution of receptive field changes in a model of peri-saccadic perception: predictive remapping and shifts towards the saccade target. Vision Res, 50: 1328 -1337. (2010).

19. Hafed, Z.M., Chen, C.Y., Tian, X. Vision, perception, and attention through the lens of microsaccades: Mechanisms and implications. Front. Syst. Neurosci, 9, 167. (2015).

20. Chen, C. Y., Hoffmann, K. P., Distler, C., Hafed, Z. M. The Foveal Visual Representation of the Primate Superior Colliculus. Current biology, 29(13), 2109_ 2119.e7. (2019).

21. Zirnsak, M., Moore, T. Saccades and shifting receptive fields: anticipating consequences or selecting targets? Trends in cognitive sciences, 18,12, 621-628. (2014).

22. Arkesteijn, K., Belopolsky, A. V., Smeets, J., Donk, M. The Limits of Predictive Remapping of Attention Across Eye Movements. Frontiers in psychology, 10, 1146. (2019).

23. Hafed, Z. M., and Clark, J. J. Microsaccades as an overt measure of covert attention shifts. Vision Res, 42(22):2533-45. (2002).

24. Hartmann, T.S., Zirnsak, M., Marquis, M., Hamker, F.H., Moore, T. Two types of receptive field dynamics in area V4 at the time of eye movements? Front. Syst. Neurosci, 11, 1-7. (2017). 
25. Andersen, R. A., Mountcastle, V. B. The influence of the angle of gaze upon the excitability of the light-sensitive neurons of the posterior parietal cortex. The Journal of Neuroscience, 3(3), 532-548. (1983).

26. Munoz, D. P., and Wurtz, R. H. Fixation cells in monkey superior colliculus. I. Characteristics of cell discharge. J. Neurophysiol. 70, 559-575. (1993).

27. Hafed, Z. M., Goffart, L., Krauzlis, R. J. A neural mechanism for microsaccade generation in the primate superior colliculus. Science, Vol. 323, No. 5916, pp. 940-943. (2009).

28. Deubel, H., Bridgeman, B., Schneider, WX. Immediate post-saccadic information mediates space constancy. Vision Res 38:3147-3159. (1998).

29. Assad, J. A., Maunsell, J. H. Neuronal correlates of inferred motion in primate posterior parietal cortex. Nature, 373(6514), 518-521.(1995).

30. Zhou, Y., Liu, Y., Lu, H., Wu, S., Zhang, M. Neuronal representation of saccadic error in macaque posterior parietal cortex (PPC). eLife, 5. (2016).

31. Connor, C. E., Gallant, J. L., Preddie, D. C., Van Essen, D. C. Responses in area V4 depend on the spatial relationship between stimulus and attention. Journal of Neurophysiology, 75(3), 1306-1308. (1996).

32. Kaiser, M., Lappe M. Perisaccadic mislocalization orthogonal to saccade direction. Neuron, 22;41(2), 293-300. (2004).

33. Zimmermann, E., Morrone, M. C., Burr, D. C. Visual mislocalization during saccades sequences. Exp Brain Res, 233(2):577-585. (2014). attentional deployment during saccadic programming. Journal of Vision, 6(3), 196-212. (2006) 
35. Montagnini A, Castet E. Spatiotemporal dynamics of visual attention during saccade preparation: Independence and coupling between attention and movement planning. $J$ Vis. 7(14):8.1-16. (2007).

36. Neupane, S., Guitton, D., and Pack, C. C. Two distinct types of remapping in primate cortical area V4. Nat. Commun.7:10402. (2016).

37. Rolfs, M., Jonikaitis, D., Deubel, H., and Cavanagh, P. Predictive remapping of attention across eye movements. Nat. Neurosci. 14, 252-256. (2011).

38. Szinte, M., Jonikaitis, D., Rangelov, D., Deubel, H. Pre-saccadic remapping relies on dynamics of spatial attention. eLife, 7:e37598. (2018).

39. Harmony, T. The functional significance of delta oscillations in cognitive processing. Frontiers in Integrative Neuroscience, 7, 83. (2013).

40. Ioannides AA, Kostopoulos GK, Liu L, Fenwick PB. MEG identifies dorsal medial brain activations during sleep. Neuroimage 44:455-468. (2009).

41. Benedetto, A., Morrone, M.C. Visual sensitivity and bias oscillate phase-locked to saccadic eye movements. J. Vis. 19, 15. (2019).

42. Benedetto, A., and Morrone, M. C. Saccadic suppression is embedded within extended oscillatory modulation of sensitivity. The Journal of Neuroscience, 37 (13), 3661-3670. (2017).

43. Hogendoorn, H. Voluntary saccadic eye movements ride the attentional rhythm. Journal of Cognitive Neuroscience, 28 (10), 1625-1635. (2016). neuronal coherence. Trends in Cognitive Sciences, 9(10), 474-480. (2005). 
46. Honda $H$. The time courses of visual mislocalization and of extraretinal eye position signals at the time of vertical saccades. Vision Res., 31(11):1915-21. (1991).

47. Porter, T. C. Contributions to the study of flicker II. Proceedings of the Royal Society of London Series A, 70, 313-329. (1902).

48. Humphries, D., Driver, P. Protean defence by prey animals. Oecologia, 5 285-302. (1970).

49. Furuichi, N. Dynamics between a predator and a prey switching two kinds of escape motions. J Theor Biol, 217(2): 159-66. (2002).

50. Botev, Z. I., Grotowski, J. F., Kroese, D. P. Kernel density estimation via diffusion. Annals of Statistics, 38:2916-2957. (2010).

51. Crossland, M.D., Sims, M., Galbraith, R.F., Rubin, G.S. Evaluation of a new quantitative technique to assess the number and extent of preferred retinal loci in macular disease. Vision Research, 44(13), 1537-1546. (2004).

52. Ludwig, C. J. H., Gilchrist, I. D. Measuring saccade curvature: A curve-fitting approach. Behavior Research Methods, Instruments, \& Computers, 34, 618-624. (2002).

53. Nandy, A. S., Nassi, J. J., Reynolds, J. H. Laminar Organization of Attentional Modulation in Macaque Visual Area V4. Neuron, 4;93(1), 235-246. (2017).

54. Tass, P., Rosenblum, M.G., Weule, J., Kurths, J., Pikovsky, A., Volkmann, J., Schnitzler, A., Freund, H. J. Detection of n:m Phase Locking from Noisy Data: Application to Magnetoencephalography. Physical review letters, 81, 3291-3294. (1998). Kernel Approach with S-Plus Illustrations (Oxford University Press, Oxford, 1997). 
bioRxiv preprint doi: https://doi.org/10.1101/2021.10.13.464140; this version posted November 19, 2021. The copyright holder for this preprint (which was not certified by peer review) is the author/funder. All rights reserved. No reuse allowed without permission.

A

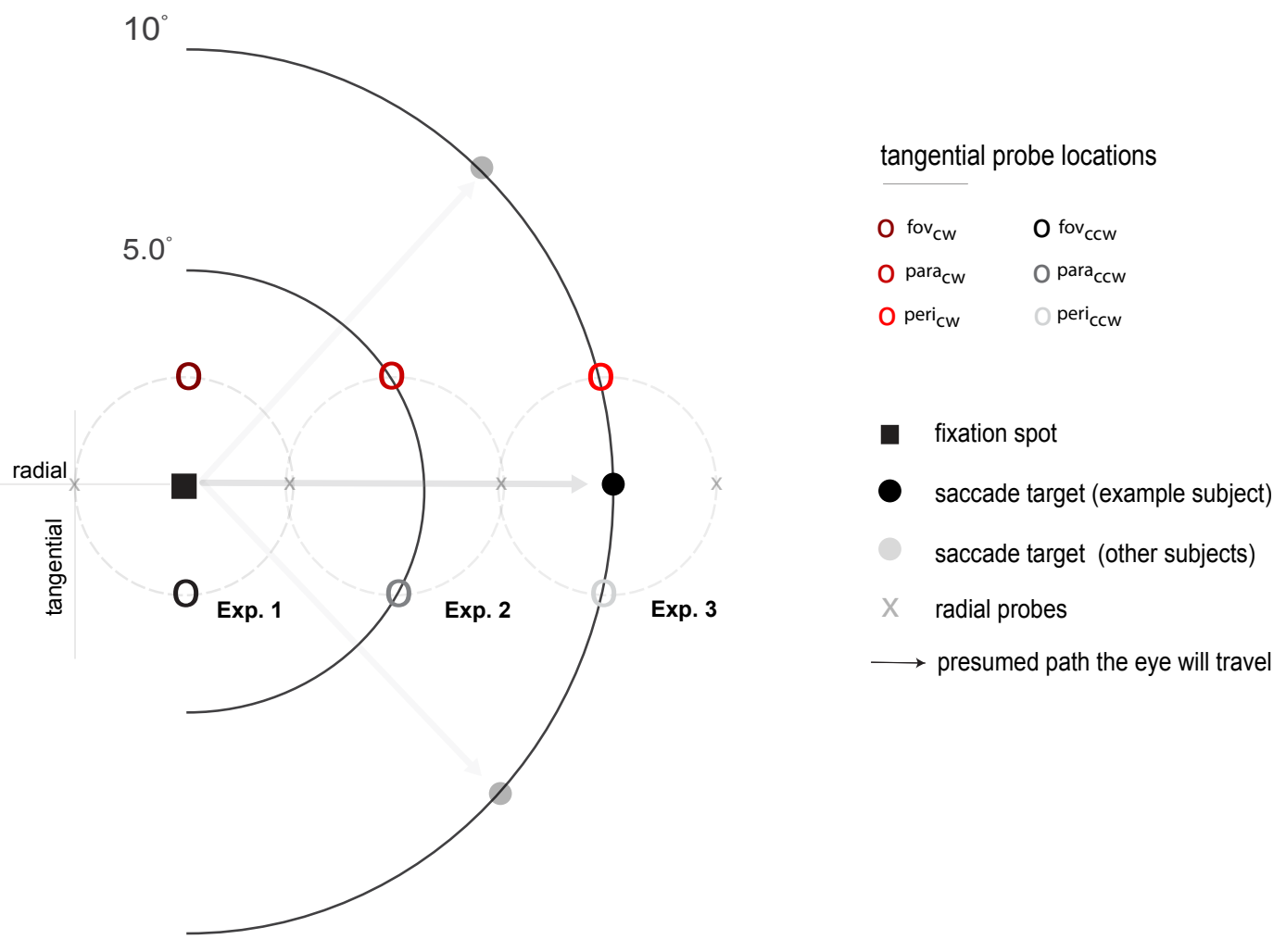

B

for illustration purposes

- fixation on

- target on central motor cue

o example probe $\left(\right.$ para $\left._{\mathrm{cw}}\right)$

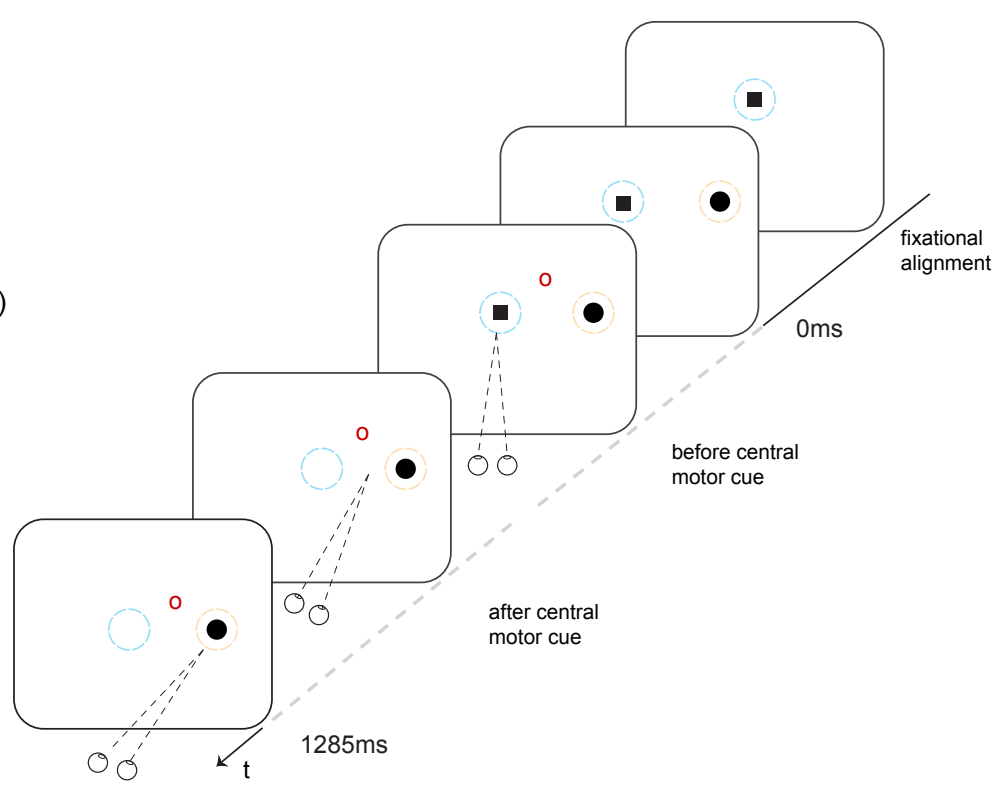

Fig 1. Behavioural protocol. (A) Markers in the illustration of visual space show all retinotopic locations at which a probe was flashed before and after the deployment of attention, saccade planning, and, finally, the execution of a saccade. Probes of interest, presented along the tangential axes, are conspicuously highlighted in open circle markers. (B) Temporal sequence of a valid trial across experiments with respect to target onset. 
bioRxiv preprint doi: https://doi.org/10.1101/2021.10.13.464140; this version posted November 19, 2021. The copyright holder for this preprint

A

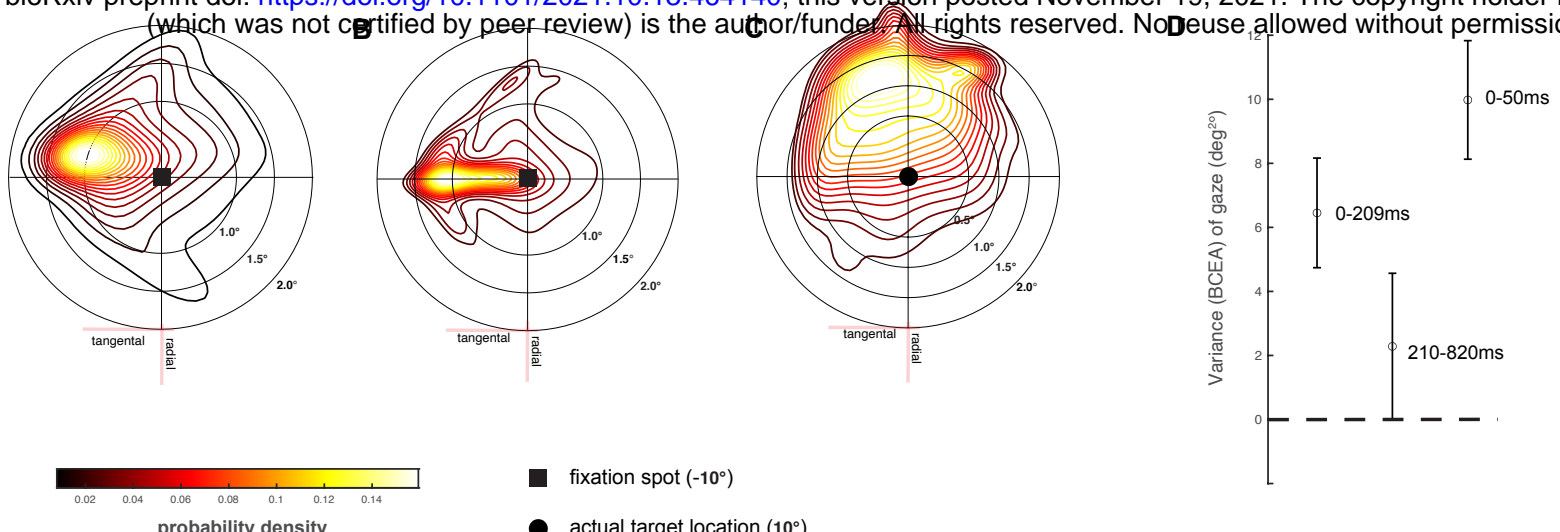

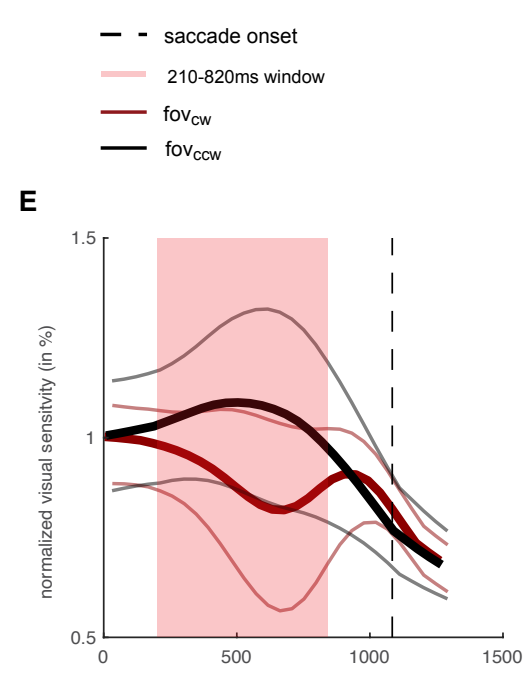

E

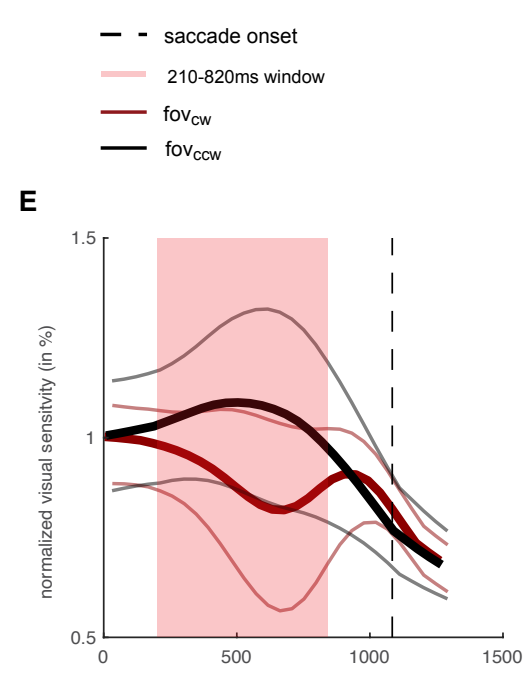

time of flash w.r.t. target onset (in ms)

- actual target location $\left(10^{\circ}\right)$
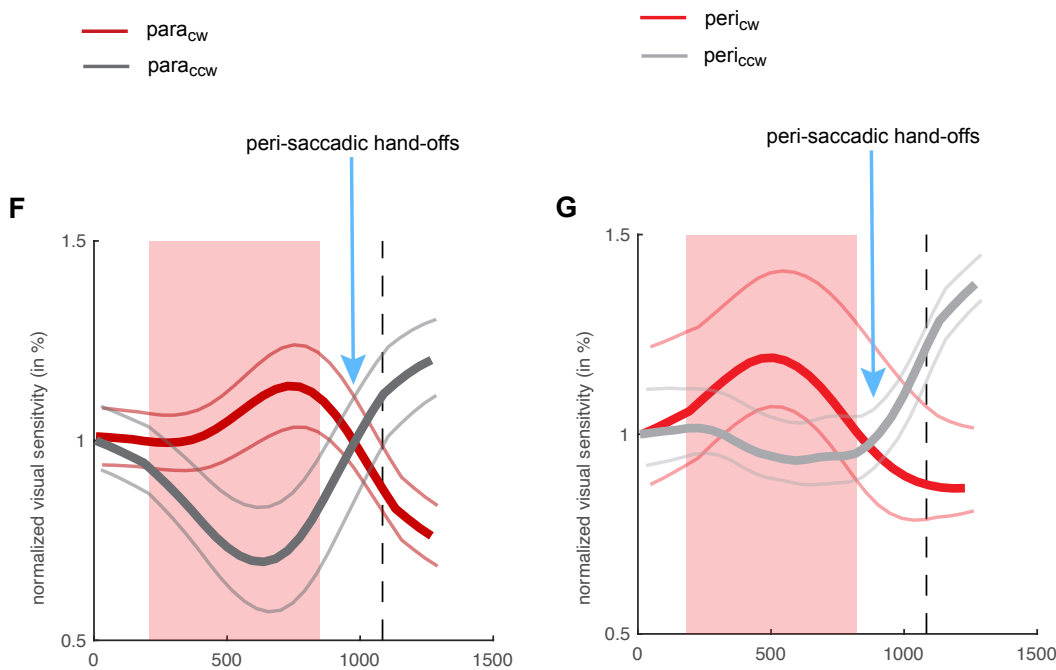

H

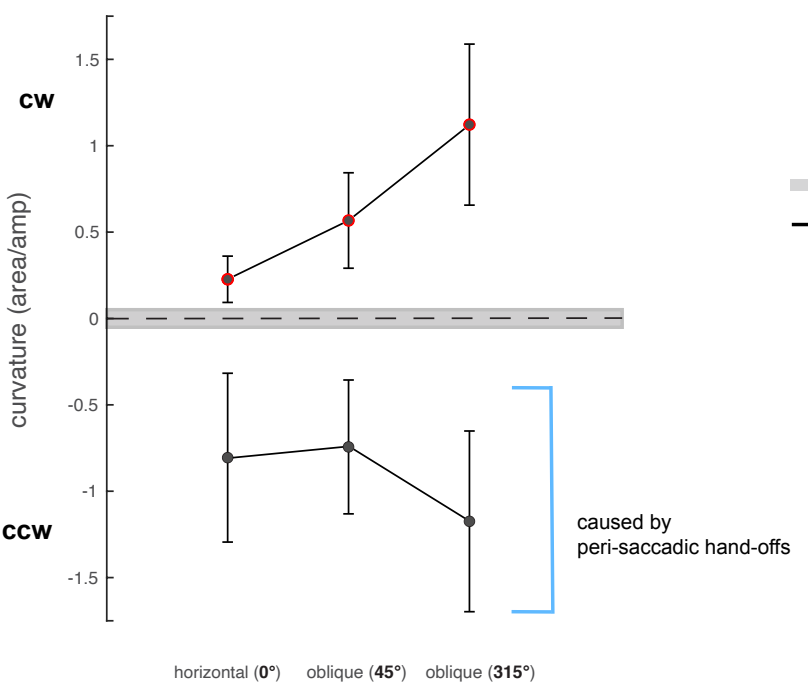

mean curvature towards $\mathrm{cw}(\mathrm{n}=\mathbf{4 3 9 0}$

mean curvature towards ccw $(n=1618)$

expected magnitude in non-curved visual space

_ saccades travel along a relatively straight line

Fig 2. Behavioural data. (A-D) Probability density map of foveal gaze and saccade landing was calculated using a bivariate gaussian kernel along with their bivariate contour ellipse area estimates for each probability density map. Error estimates are standard error of means across observers. (E-G) Shifts in visual sensitivity along tangential axes with respect to target onset; error estimates were calculated using a 20 -fold jackknife procedure. $(\mathrm{H})$ Mean saccade trajectory deviation for horizontal and oblique saccades across experiments. Positive values on the ordinate denote trajectory deviation towards a clockwise location (cw), while negative values denote trajectory deviation towards a counterclockwise location (ccw). 
bioRxiv preprint doi: https://doi.org/10.1101/2021.10.13.464140; this version posted November 19, 2021. The copyright holder for this preprint (which was not certified by peer review) is the author/funder. All rights reserved. No reuse allowed without permission.

A

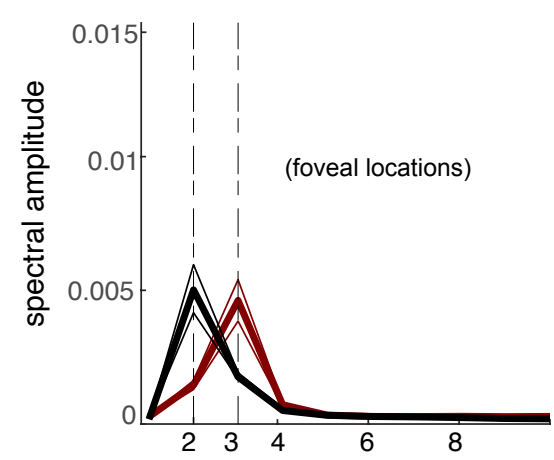

C

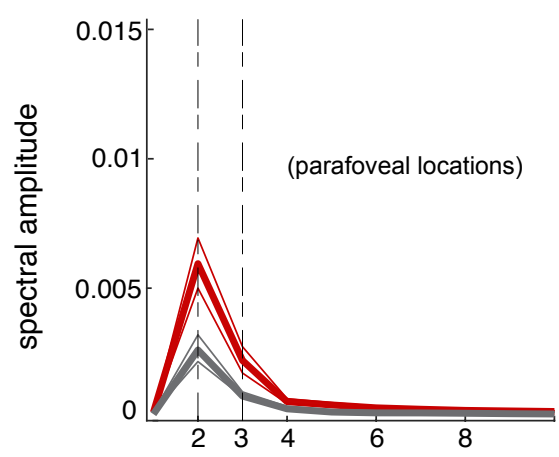

$E$

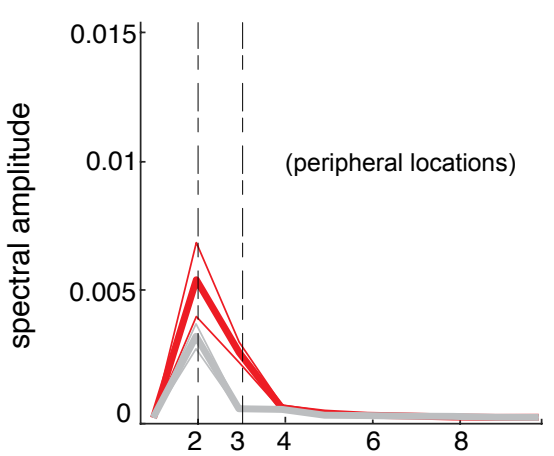

B

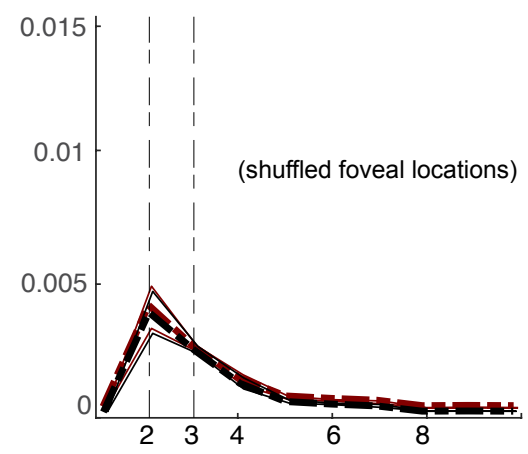

$\mathrm{D}$

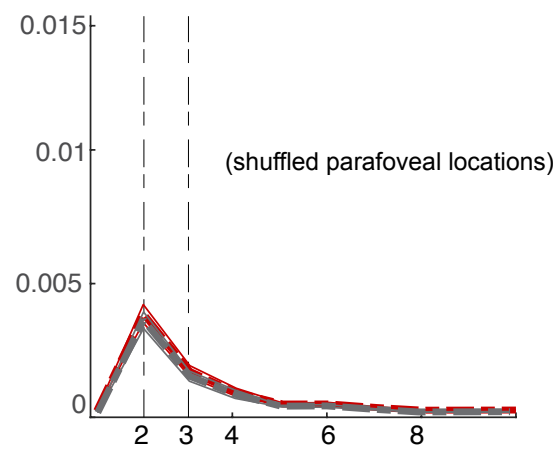

$\mathrm{F}$

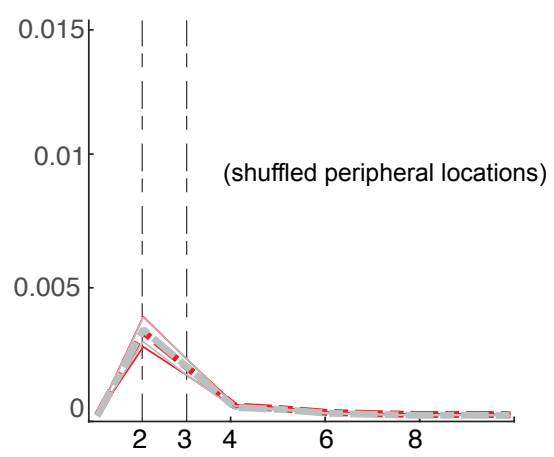

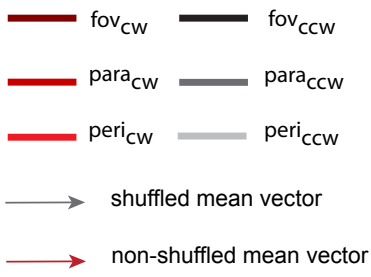

$\mathrm{G}$ cross-location phase coherence $(\sim 2 \mathrm{~Hz})$

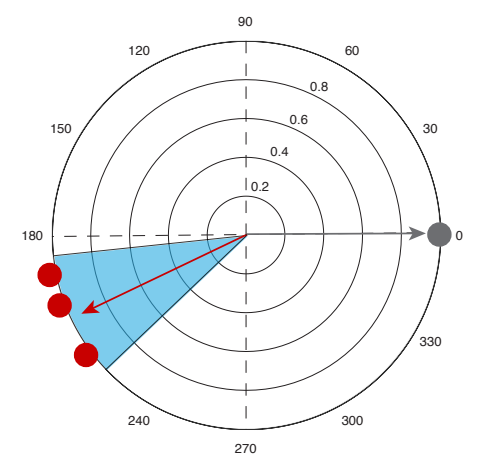

$\mathrm{H}$

cross-location phase coherence $(\sim 2 \mathrm{~Hz})$

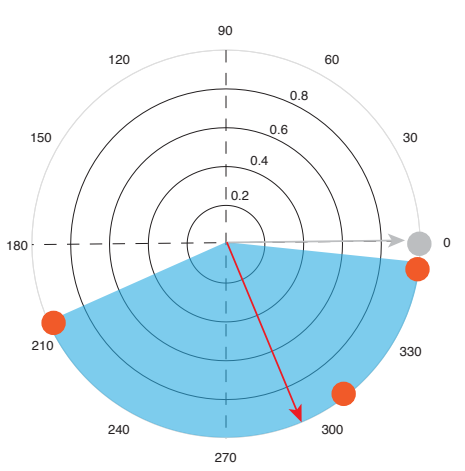

Fig 3. Frequency representation of visual sensitivity and cross-location phase coherence. (A-F) Representation of raw detrended visual sensitivity at less and more eccentric locations in visual space. Shuffled $(n=1000$, dashed line) versus unshuffled (solid line) data set were used to compute location-specific spectral signatures at a $p<0.05$. (G-H) For parafoveal and peripheral clockwise and counterclockwise locations a cross-location phase coherency analysis was performed. 


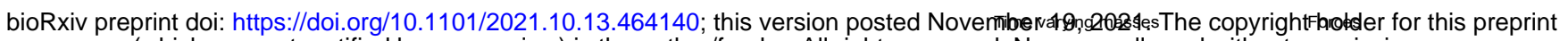
(which was not certified by peer review) is the author/funder. All rights reserved. No reuse allowed without permission.
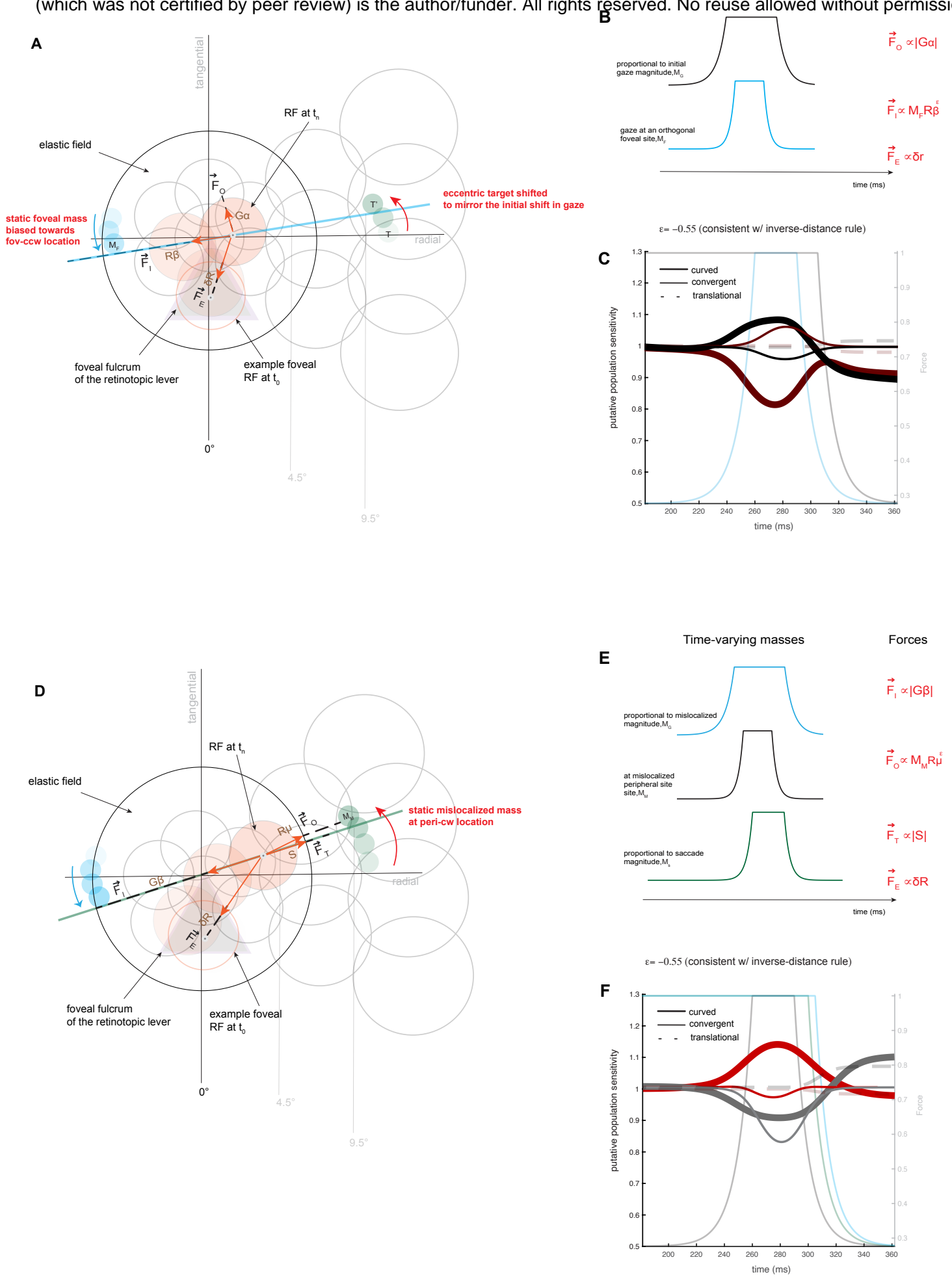

Fig 4. Model architecture and neurobiological force field with simulated putative population sensitivity. (A) For illustration purposes, a single eccentricity dependent population receptive field ( $p R F)$ within a retinotopic field is highlighted in pink. A pRF is defined by its center, size, and an eccentric dependent population elastic field (pEF). (B) MGa denotes gaze transitional signals modelled as a virtual mass at infinity located in the direction of an upper tangential location approximating the initial shift in gaze. $M_{F}$ denotes compressive signals modelled as a varying mass located at a lower tangential location in the fovea. (C) Simulation results in the foveal region when a pRF is under the influence of a resultant force exerted by (i) $M_{F}$ and $M_{G \alpha}(c u r v e d$ space, thick marker) (ii) $M_{C}$ (convergent signals modelled as a varying mass at a stationary peripheral site; see Methods) and (iii) $\mathrm{M}_{\mathrm{T}}$ (saccade transitional signals modelled as a virtual mass at infinity located in the direction of a straight saccade; see Methods). (D) A pRF bounded by pEF is highlighted in pink for parafoveal simulations. (E) MG denotes gaze translational signals modelled as a virtual mass at infinity located in the direction of a downward shifts in gaze, $M_{M}$ denotes a varying mass at a mislocalized peripheral target, and $\mathrm{M}_{\mathrm{S}}$ denotes the curved transitional signals modelled as a virtual mass at infinity located in the direction of a curvilinear saccade. (F) Curved, convergent and translational simulation results. 